\title{
NaI(Tl) Detector Efficiency Computation Using Radioactive Parallelepiped Sources Based on Efficiency Transfer Principle
}

\author{
Mohamed S. Badawi, ${ }^{1}$ Mona M. Gouda, ${ }^{1}$ Ahmed M. El-Khatib, ${ }^{1}$ Abouzeid A. Thabet, ${ }^{2}$ \\ Ahmed A. Salim, ${ }^{1}$ and Mahmoud I. Abbas ${ }^{1}$ \\ ${ }^{1}$ Physics Department, Faculty of Science, Alexandria University, Alexandria 21511, Egypt \\ ${ }^{2}$ Department of Medical Equipment Technology, Faculty of Allied Medical Sciences, Pharos University in Alexandria, \\ Alexandria 21648, Egypt
}

Correspondence should be addressed to Mohamed S. Badawi; ms241178@hotmail.com

Received 26 July 2015; Accepted 22 October 2015

Academic Editor: George Bakos

Copyright ( 2015 Mohamed S. Badawi et al. This is an open access article distributed under the Creative Commons Attribution License, which permits unrestricted use, distribution, and reproduction in any medium, provided the original work is properly cited.

The efficiency transfer (ET) principle is considered as a simple numerical simulation method, which can be used to calculate the full-energy peak efficiency (FEPE) of $3^{\prime \prime} \times 3^{\prime \prime} \mathrm{NaI}(\mathrm{Tl})$ scintillation detector over a wide energy range. In this work, the calculations of FEPE are based on computing the effective solid angle ratio between a radioactive point and parallelepiped sources located at various distances from the detector surface. Besides, the attenuation of the photon by the source-to-detector system (detector material, detector end cap, and holder material) was considered and determined. This method is straightforwardly useful in setting up the efficiency calibration curve for $\mathrm{NaI}(\mathrm{Tl})$ scintillation detector, when no calibration sources exist in volume shape. The values of the efficiency calculations using theoretical method are compared with the measured ones and the results show that the discrepancies in general for all the measurements are found to be less than $6 \%$.

\section{Introduction}

Determination of the absolute efficiency of $\mathrm{NaI}$ and $\mathrm{Ge}$ detectors has been a long standing problem in gamma-ray spectrometry and numerous reports have been published during the last decades as [1]. The calibration of gamma-ray detectors and sources is still laborious and time consuming and requires extensive operator experience [2]. Accurate calibrated photon emitting sources are difficult to prepare for all the geometrical arrangements used in gamma-ray spectroscopy and sometimes nonfeasible as, for example, in the case of metallic extended samples in neutron activation analysis [3]. In order to overcome the above problem, several nonexperimental methods have been proposed and applied, depending on the photon energy and source-detector geometry and volume [4]. An alternative possibility of being able to compute the efficiencies is thus highly desirable. One of the most common approaches, which only requires point source measurements, is called the efficiency transfer method and was pioneered by Moens et al. [5].

The efficiency transfer principle (ET) means the change in efficiency values under conditions of measurement different from those of calibration setup. It can be determined on the basis of variation of the geometrical parameters of the sourcedetector arrangement (effective solid angle ratio) [6]. In the previous group work, the efficiency transfer (ET) principle was used to determine the efficiency of the detector corresponding to different sample shapes at different distances from the detector surface [7]. The calculations of the effective solid angle ratio including the attenuation of any material between the source and the detector are based on the direct mathematical method, such as that reported in [8], which was successfully used to calibrate different detectors using different shapes radioactive sources.

In the present work, the efficiency transfer (ET) principle is used to calculate the full-energy peak efficiency (FEPE) of 
$\mathrm{NaI}(\mathrm{Tl})$ detector for axial radioactive parallelepiped sources based on the FEPE calibration for point source as a reference [9]. The calculations of the effective solid angle are based on new mathematical method reported by Hamzawy [10], where an expression to calculate the total efficiency was derivative of the cylindrical $\mathrm{NaI}(\mathrm{Tl})$ detector for using axis-off point and coaxial circular disc sources. These expressions are shorter than those in previous studies and easier to calculate, where they are in a form of an elliptical integrations type, which saves the program length and the running time and increases the accuracy of calculations [11].

\section{Mathematical Viewpoint}

The efficiency transfer principle as presented in [12] was applied to obtain the efficiency calibration curves of the gamma-ray detectors based on the following equation:

$$
\varepsilon_{\text {target }}=\frac{\Omega_{\text {target }}}{\Omega_{\text {ref }}} \varepsilon_{\text {ref }} \text {, }
$$

where $\varepsilon_{\text {ref }}$ and $\Omega_{\text {ref }}$ are the detector (FEPE) for a reference source at another position and the effective solid angle subtended by the detector surface and the reference source at that position, respectively. In order to use the efficiency transfer principle, the experimental reference efficiency, $\varepsilon_{\text {ref }}$, was essentially measured [13].

However, $\varepsilon_{\text {target }}$ and $\Omega_{\text {target }}$ are the detector (FEPE) for the target source and the effective solid angle subtended by the detector surface and the target source at that position, respectively.

Consider a cylindrical detector with radius, $R$, length, $L$, and a point source positioned at height, $h$, from the detector surface and placed at a lateral distance from the detector axis, $\rho$, smaller than the detector radius, as shown in Figure 1 .

In this case, the effective solid angle can be calculated assuming that each photon emitted from the point source will pass through the detector active volume and will traverse distance, $d$, until it emerges from the crystal. According to (2) and (3), two cases have to be considered:

(i) The photon may enter from the detector surface and emerge from the detector base, covering distance, $d_{1}$, given by

$$
d_{1}=\frac{L}{\cos \theta}
$$

(ii) The photon may enter from the detector surface and emerge from detector side, covering distance, $d_{2}$, given by

$$
d_{2}=\frac{\rho \cos \varphi+\sqrt{R^{2}-\rho^{2} \sin ^{2} \varphi}}{\sin \theta}-\frac{h}{\cos \theta} .
$$

The direction of the incident photon is defined by polar $(\theta)$ and azimuthal $(\varphi)$ angles, where the azimuthal $(\varphi)$ angle takes the value from 0 to $2 \pi$, while the polar angle $(\theta)$

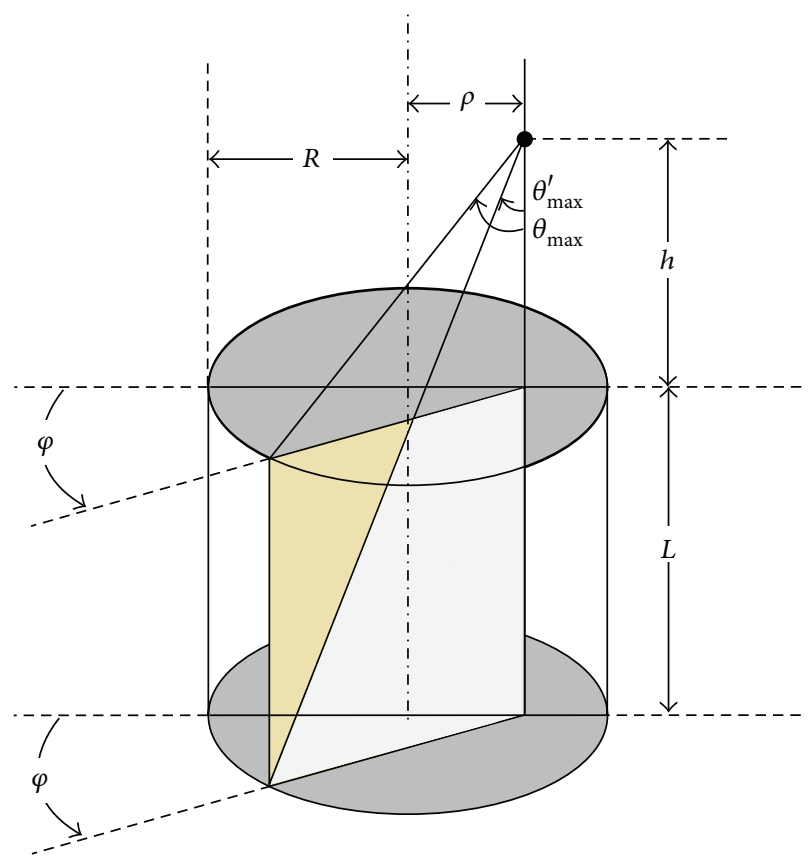

FIGURE 1: Point source configuration with $\rho \leq R$.

takes the two different steps based on the source-to-detector configuration and given by

$$
\begin{aligned}
& \theta_{\max }=\tan ^{-1}\left(\frac{\rho \cos \varphi+\sqrt{R^{2}-\rho^{2} \sin ^{2} \varphi}}{h}\right), \\
& \theta_{\max }^{\prime}=\tan ^{-1}\left(\frac{\rho \cos \varphi+\sqrt{R^{2}-\rho^{2} \sin ^{2} \varphi}}{h+L}\right),
\end{aligned}
$$

where $\theta_{\max }$ and $\theta_{\max }^{\prime}$ are the photon maximum polar angles for entering through the detector face and exiting from the detector base, respectively.

The effective solid angle, $\Omega_{\text {Eff(Non-Axial Point) }}$, in case of a nonaxial point source is given by

$$
\begin{aligned}
& \Omega_{\text {Eff(Non-Axial Point) }}=\int_{0}^{2 \pi}\left[\int_{0}^{\theta_{\max }^{\prime}} f_{\text {att }} f_{1} \sin \theta \mathrm{d} \theta\right. \\
& \left.+\int_{\theta_{\max }^{\prime}}^{\theta_{\max }} f_{\text {att }} f_{2} \sin \theta \mathrm{d} \theta\right] \mathrm{d} \varphi,
\end{aligned}
$$

where

$$
\begin{aligned}
f_{i} & =\left(1-e^{-\mu \cdot d_{i}}\right), \quad i=1,2, \\
f_{\text {att }} & =e^{-\sum_{i=1}^{n} \mu_{i} \delta_{i}}, \\
\delta_{i} & =\left(\frac{t_{i}}{\cos \theta}\right) \quad \text { For the front absorber layer, } \\
\delta_{i} & =\left(\frac{t_{i}}{\sin \theta}\right) \quad \text { For the side absorber layers, }
\end{aligned}
$$


where $\mu$ in (6) is the attenuation coefficient of the detector active material, while $f_{\text {att }}$ is the attenuation factor of the absorber layers with attenuation coefficients, $\mu_{1}, \mu_{2}, \ldots, \mu_{n}$, and with thickness, $t_{1}, t_{2}, \ldots, t_{n}$, between the source-todetector systems; also, $\delta_{i}$ is the photon path lengths inside the absorber material.
If there is a parallelepiped source with dimensions $2 a$ (length), $2 b$ (width), and $H$ (height) as illustrated in Figures 2 and 3 , the effective solid angle, $\Omega_{\mathrm{Eff}(\text { Parallelepiped) }}$, is given by

$$
\Omega_{\text {Eff(Parallelepiped) }}=\frac{4 \times\left(\int_{h_{o}}^{h_{o}+H}\left(\int_{0}^{\alpha_{T}} \int_{0}^{\rho_{1}} S_{c} S_{f} \Omega_{\text {Eff(Non-Axial Point) }} \rho \mathrm{d} \rho \mathrm{d} \alpha+\int_{\alpha_{T}}^{\pi / 2} \int_{0}^{\rho_{2}} S_{c} S_{f} \Omega_{\text {Eff(Non-Axial Point) }} \rho \mathrm{d} \rho \mathrm{d} \alpha\right) \mathrm{d} h\right)}{4 a b H},
$$

where

$$
\begin{aligned}
\alpha & =\tan ^{-1}\left(\frac{y}{x}\right), \\
\alpha_{T} & =\tan ^{-1}\left(\frac{b}{a}\right), \\
\rho_{1} & =\frac{a}{\cos \alpha}, \\
\rho_{2} & =\frac{b}{\sin \alpha},
\end{aligned}
$$

while $S_{f}$ and $S_{c}$ are the self-attenuation factor of the source matrix and the attenuation factor of the container material, respectively, which can be represented by the following equations:

$$
\begin{aligned}
& S_{f}=e^{-\mu_{s} t_{i}}, \\
& S_{c}=e^{-\mu_{c} t_{c i}},
\end{aligned}
$$

where $\mu_{s}$ is the attenuation coefficient of the source matrix, where the source is filled with radioactive material, and $\mu_{c}$ is the attenuation coefficient of the source container material, while $t_{i}$ is the distance traveled by the emitted photon inside the source and $t_{c i}$ is the distance traveled by the emitted photon inside the source container material.

The container wall thicknesses are $\Delta h, \Delta y$, and $\Delta x$ for the bottom, width, and length, respectively. Table 1 shows the possible path lengths traveled by the photon within the source matrix and the source container material, while Table 2 shows the values of the polar and azimuthal angles of the source matrix and the source container material.

The distance traveled inside the source matrix, $t_{i}$, varies upon the variation of the polar and azimuthal angles $(\theta, \varphi)$ inside the source itself, such as what is given in Table 3.

The distance traveled inside the source container material, $t_{c i}$, varies upon the variation of the polar and azimuthal angles $(\theta, \varphi)$ inside the source itself and can be given in Table 4.

A computer program has been designed to calculate the effective solid angle ratio between point and parallelepiped sources located at various distances from the detector surface. The numerical computation of the double integrals is performed using the trapezoidal rule. Moreover, the accuracy of the integration increases by increasing the number of integration steps, $n$. It was stated that integration converges very well at $n=40$.

The full-energy peak efficiency (FEPE) can be calculated based on the reference measured full-energy peak efficiency [14] by the following formula:

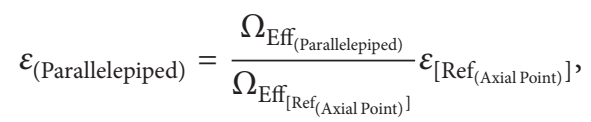

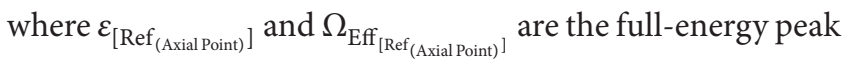
efficiency (FEPE) of the $\mathrm{NaI}(\mathrm{Tl})$ detector and the effective solid angles subtended by the detector surface for using an axial situated point source located at reference position from the detector surface, respectively. In this case, to obtain

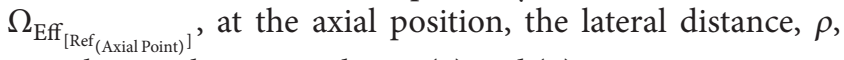
must be equal to zero value in (3) and (4).

However, $\varepsilon_{\text {(Parallelepiped) }}$ and $\Omega_{\mathrm{Eff}_{\text {(Parallelepiped) }}}$ are the fullenergy peak efficiency (FEPE) of the $\mathrm{NaI}(\mathrm{Tl})$ detector and the effective solid angles subtended by the detector surface for using radioactive parallelepiped source located at any axial position different from the reference one, respectively.

\section{Experimental Setup}

The detector used in these measurements is $\mathrm{NaI}(\mathrm{Tl})$ detector (CANBERRA-802 series). The nominal dimensions of the detector crystal are $76.2 \mathrm{~mm}$ diameter and $76.2 \mathrm{~mm}$ length. It is mounted inside a protective aluminum end cap $(0.5 \mathrm{~mm}$ thick, mass per unit area $147 \mathrm{mg} / \mathrm{cm}^{2}$ ) with its axis being vertical, reflector-oxide $(1.6 \mathrm{~mm}$ thick, mass per unit area $88 \mathrm{mg} / \mathrm{cm}^{2}$ ), magnetic/light shield-conetic lined steel (typical operating voltages: cathode to anode $+800 \mathrm{~V} \mathrm{dc}$, dynode to dynode $+80 \mathrm{~V} \mathrm{dc}$, and cathode to dynode $+150 \mathrm{~V} \mathrm{dc}$ ). The detector relative energy resolution is $8.5 \%$ at $662 \mathrm{keV}$. The signals from the detector were amplified and shaped by Osprey Universal Digital MCA Tube Base for Scintillation Spectrometry. The Osprey MCA designed to be temperature stabilized with $\mathrm{NaI}$ detector, standard with 14 pins using 10stage PMTs, high performance, high-voltage power supply (HVPS), preamplifier, fully integrated MCA includes all needs to support spectrometry system for working in both laboratory and field. The MCA can be controlled through the USB cable to control the display, acquisition, and analysis process with the Genie 2000 software [15]. 
TABLE 1: The possible path lengths traveled by the photon within the source matrix and the source container material.

Source matrix

Polar angles

Azimuthal angles

$\theta_{1}=\tan ^{-1}\left|\frac{b+y}{\left(h-h_{o}\right) \sin \beta}\right|$

$\varphi_{1}=\frac{\pi}{2}-\alpha+\tan ^{-1}\left(\frac{a-x}{b+y}\right)$

$\theta_{2}=\tan ^{-1}\left|\frac{a-x}{\left(h-h_{o}\right) \cos \beta}\right|$

$\varphi_{2}=\pi-\alpha+\tan ^{-1}\left(\frac{b-y}{a-x}\right)$

$\theta_{3}=\tan ^{-1}\left|\frac{b-y}{\left(h-h_{o}\right) \sin \beta}\right|$

$\varphi_{3}=2 \pi-\alpha-\tan ^{-1}\left(\frac{b-y}{a+x}\right)$

$\theta_{4}=\tan ^{-1}\left|\frac{a+x}{\left(h-h_{o}\right) \cos \beta}\right|$

$\varphi_{4}= \begin{cases}2 \pi-\alpha+\tan ^{-1}\left(\frac{b+y}{a+x}\right) & \left(\alpha \geq \alpha_{T}\right) \\ \tan ^{-1}\left(\frac{b+y}{a+x}\right)-\alpha & \left(\alpha<\alpha_{T}\right)\end{cases}$

Source container material

Polar angles

Polar angles

$\theta_{1 c}=\tan ^{-1}\left|\frac{b+\Delta y+y}{\left(h-h_{o}\right) \sin \beta}\right|$

$\varphi_{1 c}=\frac{\pi}{2}-\alpha+\tan ^{-1}\left(\frac{a+\Delta x-x}{b+\Delta y+y}\right)$

$\theta_{2 c}=\tan ^{-1}\left|\frac{a+\Delta x-x}{\left(h-h_{o}\right) \cos \beta}\right|$

$\varphi_{2 c}=\pi-\alpha+\tan ^{-1}\left(\frac{b+\Delta y-y}{a+\Delta x-x}\right)$

$\theta_{3 c}=\tan ^{-1}\left|\frac{b+\Delta y-y}{\left(h-h_{o}\right) \sin \beta}\right|$

$\varphi_{3 c}=2 \pi-\alpha-\tan ^{-1}\left(\frac{b+\Delta y-y}{a+\Delta x+x}\right)$

$\theta_{4 c}=\tan ^{-1}\left|\frac{a+\Delta x+x}{\left(h-h_{o}\right) \cos \beta}\right|$

$\varphi_{4 c}= \begin{cases}2 \pi-\alpha+\tan ^{-1}\left(\frac{b+\Delta y+y}{a+\Delta x+x}\right) & \left(\alpha \geq \alpha_{T}\right) \\ \tan ^{-1}\left(\frac{b+\Delta y+y}{a+\Delta x+x}\right)-\alpha & \left(\alpha<\alpha_{T}\right)\end{cases}$

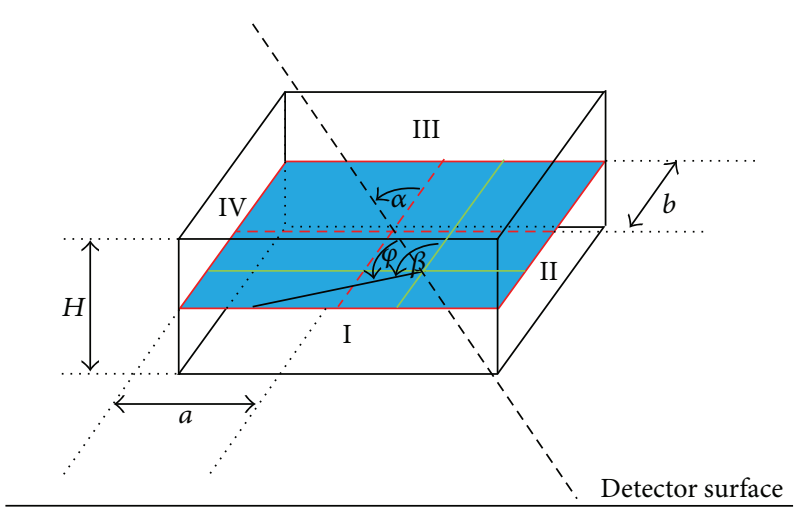

FIGURE 2: Schematic diagram of a parallelepiped source.

Two types of sources were used in the present experiment: standard point sources for the reference calibration and volume source as the target as of this study. The sources activities, their uncertainties, half-lives, photon energies, and photon emission probabilities per decay for all radionuclides used in the calibration process were listed in Tables 5(a) and 5(b).

The standard radioactive sources were purchased from Physikalisch-Technische Bundesanstalt (PTB). The radioactive substance is a very thin, compact grained layer applied to a circular area about $5 \mathrm{~mm}$ in diameter, in the middle of the source between two polyethylene foils each having a

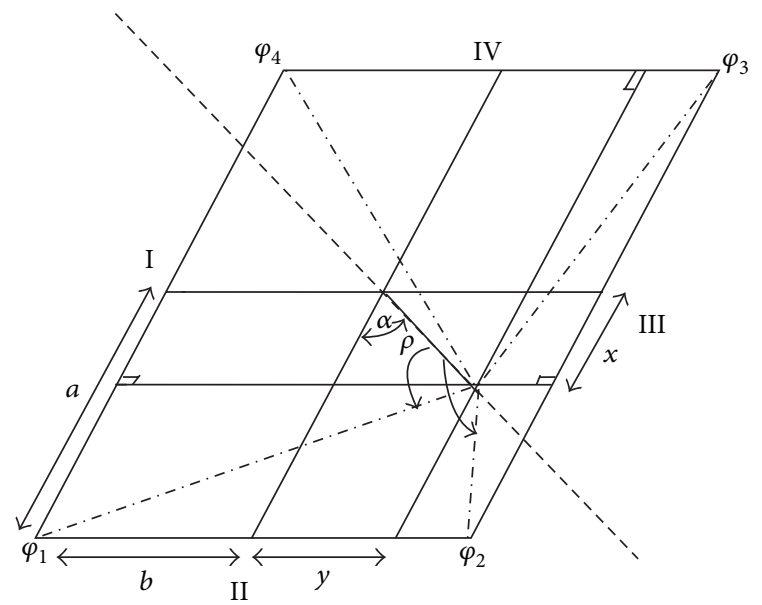

FIGURE 3: Schematic view for the azimuthal angle values in the bar source.

mass per unit area of $21.3 \pm 1.8 \mathrm{mg} \cdot \mathrm{cm}^{-2}$. By heating under pressure, the two foils are welded together over the whole area so that they are leak-proofed. To facilitate handling, the foil $26 \mathrm{~mm}$ in diameter is mounted in a circular aluminum ring (outer diameter: $30 \mathrm{~mm}$, height: $3 \mathrm{~mm}$ ) from which it can easily be removed if and when required. There are two types of the standard radioactive sources used in the present work; the first type was in the form of five point sources. These 
TABLE 2: The values of the polar and the azimuthal angles of the source matrix and the source container material.

\begin{tabular}{lll}
\hline \multirow{2}{*}{ Possible cases } & \multicolumn{2}{c}{ Possible path lengths } \\
\hline Exit from the base & $t_{0}=\frac{h-h_{0}}{\cos \theta} \quad t_{c o}=\frac{\Delta h}{\cos \theta}$ \\
\hline Exit from side I & $t_{1}=\frac{b+y}{\sin \theta \sin \beta}$ & $t_{c 1}=\frac{\Delta y}{\sin \theta \sin \beta}$ \\
\hline Exit from side II & $t_{2}=\left|\frac{a-x}{\sin \theta \cos \beta}\right|$ & $t_{c 2}=\left|\frac{\Delta x}{\sin \theta \cos \beta}\right|$ \\
\hline Exit from side III & $t_{3}=\left|\frac{b-y}{\sin \beta \sin \theta}\right|$ & $t_{c 3}=t_{c 1}=\frac{\Delta y}{\sin \theta \sin \beta}$ \\
\hline Exit from side IV & $t_{4}=\frac{a+x}{\sin \theta \cos \beta}$ & $t_{c 4}=t_{c 2}=\left|\frac{\Delta x}{\sin \theta \cos \beta}\right|$ \\
\hline where $\beta=\alpha+\varphi, x=\rho \cos \alpha, y=\rho \sin \alpha$, and $\alpha=\tan (y / x)$ \\
\hline
\end{tabular}

TABLE 3: The possible path lengths inside the source matrix.

\begin{tabular}{lcc}
\hline $\begin{array}{l}\text { Azimuthal angles } \\
\text { conditions }\end{array}$ & $\begin{array}{c}\text { Polar angles } \\
\text { conditions }\end{array}$ & Possible path lengths \\
\hline$\left(\varphi_{1} \leq \varphi<\varphi_{2}\right)$ & $\left(\theta \leq \theta_{1}\right)$ & $t_{o}$ \\
$\left(\theta_{1}<\theta \leq \theta_{\max }\right)$ & $t_{1}$ \\
$\left(\varphi_{2} \leq \varphi<\varphi_{3}\right)$ & $\left(\theta \leq \theta_{2}\right)$ & $t_{o}$ \\
\hline$\left(\varphi_{3} \leq \varphi<\varphi_{4}\right)$ & $\left(\theta \leq \theta_{3}\right)$ & $t_{2}$ \\
\hline$\left(\varphi_{4} \leq \varphi<\varphi_{1}\right)$ & $\left(\theta_{3}<\theta \leq \theta_{\max }\right)$ & $t_{o}$ \\
& $\left(\theta \leq \theta_{4}\right)$ & $t_{3}$ \\
\hline
\end{tabular}

TABLE 4: The possible path lengths inside the source container material.

\begin{tabular}{lcc}
\hline $\begin{array}{l}\text { Azimuthal angles } \\
\text { conditions }\end{array}$ & $\begin{array}{c}\text { Polar angles } \\
\text { conditions }\end{array}$ & Possible path lengths \\
\hline$\left(\varphi_{1 c} \leq \varphi<\varphi_{2 c}\right)$ & $\left(\theta \leq \theta_{1 c}\right)$ & $t_{c o}$ \\
\hline$\left(\varphi_{2 c} \leq \varphi<\varphi_{3 c}\right)$ & $\left(\theta \leq \theta_{2 c}\right)$ & $t_{c 1}$ \\
\hline$\left(\varphi_{3 c} \leq \varphi<\varphi_{4 c}\right)$ & $\left(\theta_{2 c}<\theta \leq \theta_{\max }\right)$ & $t_{c o}$ \\
\hline$\left(\varphi_{4 c} \leq \varphi<\varphi_{1 c}\right)$ & $\left(\theta_{3 c}<\theta \leq \theta_{\max }\right)$ & $t_{c 2}$ \\
\hline
\end{tabular}

sources were measured using a homemade Plexiglas holder at 3 different axial distances from the detector surface $(\mathrm{P} 4=$ $20 \mathrm{~cm}, \mathrm{P} 6=30 \mathrm{~cm}$, and $\mathrm{P} 8=40 \mathrm{~cm}$ ) and start from P4, while the second type of radioactive sources is parallelepiped which contains ${ }^{152} \mathrm{Eu}$ solution. These radioactive parallelepiped sources were measured at two positions, on the Plexiglas holder base with thickness $0.363 \mathrm{~cm}$ at position P0 "placed directly on the detector end cap" and at position P1 $=5 \mathrm{~cm}$ from the detector surface.

\section{Results and Discussion}

In these measurements of low activity sources, the dead time was always less than $3 \%$. So the corresponding factor is obtained simply using ADC live time. The peak areas, live time, real time, and starting time for each spectrum were entered in the spreadsheet used to calculate the efficiency curves [15]. Once the full-energy peak area is obtained, the full-energy peak efficiency values can be calculated by the following equation:

$$
\varepsilon(E)=\frac{N(E)}{T A_{s} P(E)} \prod C_{i}
$$

where $\varepsilon(E)$ is the full-energy peak efficiency as a function of the photon energy, $N(E)$ is the net peak area, $A_{s}$ is the radionuclide activity at the time of standardization (Bq), $T$ is the live time (s), $P(E)$ is the photon emission intensity at energy $E$, and $C_{i}$ are the correction factors due to dead time and radionuclide decay [15]. The experimental uncertainties on acquisition time and source distance were considered negligible; thus, the standard deviation on $\varepsilon, \sigma_{\varepsilon}$, was determined by the uncertainties on $N(E), P(E)$, and $A_{s}$ and was calculated according to the propagation of error law according to the following equation:

$$
\sigma_{\varepsilon}=\varepsilon \sqrt{\left(\frac{\partial \varepsilon}{\partial A}\right)^{2} \sigma_{A}^{2}+\left(\frac{\partial \varepsilon}{\partial P}\right)^{2} \sigma_{P}^{2}+\left(\frac{\partial \varepsilon}{\partial N}\right)^{2} \sigma_{N}^{2}}
$$

The relative uncertainty in the peak area was always arranged to be $0.5 \%$ and so the absolute uncertainty measurements were dominated by the uncertainty in the radionuclide activity. Uncertainties in the relative efficiency measurements with a given source as a function of distance were generally determined by a combination of counting statistics. Once the individual efficiencies have been obtained by applying the correction factors, the overall efficiency curve is obtained by fitting the experimental points to a polynomial logarithmic function of the fourth order using nonlinear least square fit. In this way, the correlation between data points from the same calibrated source has been included to avoid the overestimation of the uncertainty in the measured efficiency.

In the present work, the analytical formulae are considering the detector active volume and the effective geometrical solid angle, corresponding to a radioactive point and parallelepiped sources located at various distances from the detector surface in order to obtain a simple formula for the efficiency transfer method. In addition, the self-attenuation coefficient of the source matrix, the attenuation factors of the source container, and the detector housing materials are also treated by calculating the path length within these materials.

The reference experimental calibration curve was obtained by using radioactive point sources measured at three different axial distances from the detector surface, P4, P6, and P8, as represented in Figure 4. The efficiency transfer principle is used to convert the full-energy peak efficiency curve from these positions' "reference curve" to the other (FEPE), which was located at positions P0 up to P1 for using radioactive parallelepiped sources V1 and V2 based 
TABLE 5: The point and volume source's description.

(a)

\begin{tabular}{|c|c|c|c|c|c|}
\hline \multicolumn{6}{|c|}{ Point source's description } \\
\hline PTB-Nuclide & $\begin{array}{c}\text { Energy } \\
(\mathrm{keV})\end{array}$ & $\begin{array}{c}\text { Emission } \\
\text { probability \% }\end{array}$ & $\begin{array}{c}\text { Half-life } \\
\text { (days) }\end{array}$ & $\begin{array}{c}\text { Activity }(\mathrm{kBq}) \\
\text { 1 June } 2009\end{array}$ & $\begin{array}{c}\text { Uncertainty } \\
(\mathrm{kBq})\end{array}$ \\
\hline${ }^{241} \mathrm{Am}$ & 59.52 & 35.9 & 157861.05 & 259.0 & \pm 2.6 \\
\hline${ }^{133} \mathrm{Ba}$ & 80.99 & 34.1 & 3847.91 & 275.3 & \pm 2.8 \\
\hline \multirow{6}{*}{${ }^{152} \mathrm{Eu}$} & 121.78 & 28.4 & \multirow{6}{*}{4943.29} & \multirow{6}{*}{290.0} & \multirow{6}{*}{ \pm 4.0} \\
\hline & 244.69 & 7.49 & & & \\
\hline & 344.28 & 26.6 & & & \\
\hline & 778.90 & 12.96 & & & \\
\hline & 964.13 & 14.0 & & & \\
\hline & 1408.01 & 20.87 & & & \\
\hline${ }^{137} \mathrm{Cs}$ & 661.66 & 85.21 & 11004.98 & 385.0 & \pm 4.0 \\
\hline \multirow{2}{*}{${ }^{60} \mathrm{Co}$} & 1173.23 & 99.90 & \multirow{2}{*}{1925.31} & \multirow{2}{*}{212.1} & \multirow{2}{*}{ \pm 1.5} \\
\hline & 1332.5 & 99.98 & & & \\
\hline
\end{tabular}

(b)

\begin{tabular}{|c|c|c|c|}
\hline \multicolumn{4}{|c|}{ Volume source's description $(\mathrm{mm})$ and activities, $5.05 \pm 0.05 \mathrm{kBq}$} \\
\hline $\begin{array}{l}\text { Fisher Scientific Company } \\
\text { Polypropylene (PP) Parallelepiped }\end{array}$ & $\begin{array}{l}\text { Cross section } \\
\text { dimensions }\end{array}$ & $\begin{array}{c}\text { Radioactive PTB }{ }^{152} \text { Eu solution } \\
\text { height }\end{array}$ & $\begin{array}{c}\text { Thickness } \\
\text { [bottom and } \\
\text { side] }\end{array}$ \\
\hline V1 (125 mL) filled with $100 \mathrm{~mL}$ & $59.23 \times 37.88$ & 51.02 & 1.50 \\
\hline V2 $(250 \mathrm{~mL})$ filled with $200 \mathrm{~mL}$ & $60.48 \times 60.48$ & 61.40 & 1.52 \\
\hline
\end{tabular}

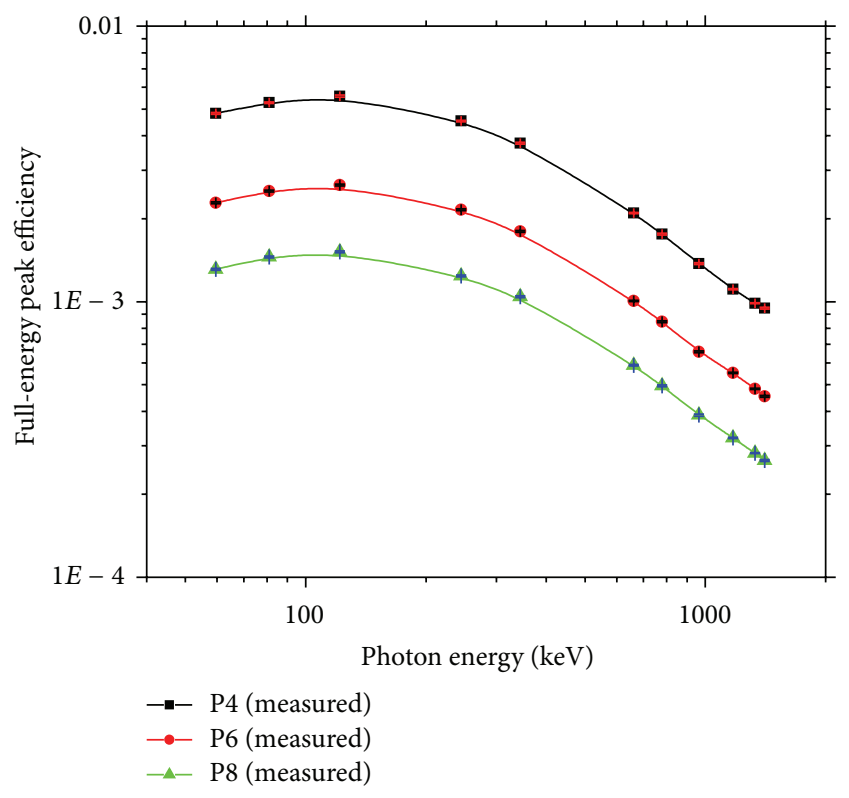

FIGURE 4: Reference experimental calibration curve for point sources measured at three different axial distances, P4, P6, and P8, from the $\mathrm{NaI}(\mathrm{Tl})$ detector surface.

on (12). In addition, the values of the effective solid angle are tabulated in Table 6 for a point and parallelepiped sources at the measurement positions. The relative deviation between the measured and the calculated efficiencies (FEPE) is given by the following equation:

$$
\Delta \%=\frac{\varepsilon_{\mathrm{Cal}}-\varepsilon_{\text {meas }}}{\varepsilon_{\text {meas }}} \times 100,
$$

where $\varepsilon_{\text {Cal }}$ and $\varepsilon_{\text {meas }}$ are the calculated and experimental efficiencies, respectively.

The FEPE values with their associated uncertainties for parallelepiped sources, V1 and V2, were measured at positions $\mathrm{P} 0$ and $\mathrm{P} 1$. These data are compared with the calculated values based on the conversion process by (12) from the reference efficiency curve for a point source at three different axial distances from the detector surface, and the comparisons are tabulated in Tables 7 and 8. In addition, the deviation percentage $\Delta \%$, between the measured and the calculated (FEPE) values at various positions is listed in the same tables.

\section{Conclusion}

This work provides an analytical method in gamma-ray spectrometry field and consider as useful tools of $\mathrm{NaI}(\mathrm{Tl})$ efficiency computation for laboratory routine measurements, saving the time and avoiding the experimental calibration process for different sample geometries when standard radioactive sources do not exist. Therefore, this method is sufficient for most routine measurement work under one 
TABLE 6: Effective solid angle for point and parallelepiped sources at various positions.

\begin{tabular}{|c|c|c|c|c|c|c|c|c|}
\hline \multirow{3}{*}{ Nuclide } & \multirow{3}{*}{$\begin{array}{c}\text { Energy } \\
\text { keV }\end{array}$} & \multicolumn{7}{|c|}{ Effective solid angle } \\
\hline & & \multicolumn{3}{|c|}{ Point sources } & \multicolumn{4}{|c|}{ Parallelepiped sources } \\
\hline & & $\left(\Omega_{\mathrm{P} 4}\right)$ & $\left(\Omega_{\mathrm{P} 6}\right)$ & $\left(\Omega_{\mathrm{P} 8}\right)$ & $\Omega_{(\mathrm{V} 1-\mathrm{P} 0)}$ & $\Omega_{(\mathrm{V} 2-\mathrm{P} 0)}$ & $\Omega_{(\mathrm{V} 1-\mathrm{P} 1)}$ & $\Omega_{(\mathrm{V} 2-\mathrm{P} 1)}$ \\
\hline Am-241 & 59.52 & $4.59 E-02$ & $2.13 E-02$ & $1.21 E-02$ & $5.59 E-01$ & $4.21 E-01$ & $1.79 E-01$ & $1.64 E-01$ \\
\hline Ba-133 & 80.99 & $5.00 E-02$ & $2.33 E-02$ & $1.32 E-02$ & $6.27 E-01$ & $4.75 E-01$ & $1.99 E-01$ & $1.82 E-01$ \\
\hline $\mathrm{Eu}-152$ & 121.78 & $5.26 E-02$ & $2.46 E-02$ & $1.40 E-02$ & $6.77 E-01$ & $5.16 E-01$ & $2.12 E-01$ & $1.96 E-01$ \\
\hline Eu-152 & 244.69 & $5.27 E-02$ & $2.52 E-02$ & $1.45 E-02$ & $6.63 E-01$ & $5.09 E-01$ & $2.03 E-01$ & $1.91 E-01$ \\
\hline $\mathrm{Eu}-152$ & 344.28 & $5.10 E-02$ & $2.46 E-02$ & $1.43 E-02$ & $6.21 E-01$ & $4.80 E-01$ & $1.91 E-01$ & $1.81 E-01$ \\
\hline Cs-137 & 661.66 & $4.66 E-02$ & $2.28 E-02$ & $1.33 E-02$ & $5.51 E-01$ & $4.31 E-01$ & $1.71 E-01$ & $1.65 E-01$ \\
\hline $\mathrm{Eu}-152$ & 778.90 & $4.54 E-02$ & $2.23 E-02$ & $1.30 E-02$ & $5.36 E-01$ & $4.21 E-01$ & $1.67 E-01$ & $1.61 E-01$ \\
\hline $\mathrm{Eu}-152$ & 964.13 & $4.38 E-02$ & $2.15 E-02$ & $1.26 E-02$ & $5.16 E-01$ & $4.07 E-01$ & $1.61 E-01$ & $1.56 E-01$ \\
\hline Co-60 & 1173.23 & $4.22 E-02$ & $2.08 E-02$ & $1.21 E-02$ & $4.97 E-01$ & $3.93 E-01$ & $1.55 E-01$ & $1.50 E-01$ \\
\hline Co- 60 & 1332.51 & $4.12 E-02$ & $2.03 E-02$ & $1.19 E-02$ & $4.85 E-01$ & $3.84 E-01$ & $1.52 E-01$ & $1.47 E-01$ \\
\hline Eu-152 & 1408.01 & $4.08 E-02$ & $2.01 E-02$ & $1.18 E-02$ & $4.80 E-01$ & $3.81 E-01$ & $1.50 E-01$ & $1.46 E-01$ \\
\hline
\end{tabular}

TABLE 7: Comparison between the measured and calculated full-energy peak efficiencies for parallelepiped sources, V1 and V2, at position P0.

\begin{tabular}{|c|c|c|c|c|c|c|c|c|c|}
\hline \multirow{2}{*}{ Nuclide } & \multirow{2}{*}{$\begin{array}{c}\text { Energy } \\
\text { keV }\end{array}$} & \multicolumn{4}{|c|}{$\varepsilon_{\mathrm{P} 4} \rightarrow \varepsilon_{(\mathrm{V} 1-\mathrm{P} 0)}$} & \multicolumn{4}{|c|}{$\varepsilon_{\mathrm{P} 4} \rightarrow \varepsilon_{(\mathrm{V} 2-\mathrm{P} 0)}$} \\
\hline & & Calculated & Measured & Unc. & $\Delta \%$ & Calculated & Measured & Unc. & $\Delta \%$ \\
\hline \multirow{6}{*}{$\mathrm{Eu}-152$} & 121.78 & $7.18 E-02$ & $7.08 E-02$ & $5.72 E-04$ & -1.43 & $5.47 E-02$ & $5.48 E-02$ & $4.41 E-04$ & 0.22 \\
\hline & 244.69 & $5.69 E-02$ & $5.60 E-02$ & $4.64 E-04$ & -1.60 & $4.37 E-02$ & $4.38 E-02$ & $3.20 E-04$ & 0.28 \\
\hline & 344.28 & $4.57 E-02$ & $4.53 E-02$ & $3.49 E-04$ & -0.77 & $3.53 E-02$ & $3.52 E-02$ & $2.66 E-04$ & -0.36 \\
\hline & 778.90 & $2.08 E-02$ & $2.09 E-02$ & $1.71 E-04$ & 0.70 & $1.63 E-02$ & $1.64 E-02$ & $1.22 E-04$ & 0.69 \\
\hline & 964.13 & $1.62 E-02$ & $1.63 E-02$ & $1.38 E-04$ & 0.42 & $1.28 E-02$ & $1.28 E-02$ & $9.85 E-05$ & 0.50 \\
\hline & 1408.01 & $1.12 E-02$ & $1.13 E-02$ & $8.65 E-05$ & 1.44 & $8.84 E-03$ & $8.85 E-03$ & $6.48 E-05$ & 0.09 \\
\hline \multirow{2}{*}{ Nuclide } & Energy & \multicolumn{4}{|c|}{$\varepsilon_{\mathrm{P} 6} \rightarrow \varepsilon_{(\mathrm{V} 1-\mathrm{P} 0)}$} & \multicolumn{4}{|c|}{$\varepsilon_{\mathrm{P} 6} \rightarrow \varepsilon_{(\mathrm{V} 2-\mathrm{P} 0)}$} \\
\hline & $\mathrm{keV}$ & Calculated & Measured & Unc. & $\Delta \%$ & Calculated & Measured & Unc. & $\Delta \%$ \\
\hline \multirow{6}{*}{ Eu-152 } & 121.78 & $7.31 E-02$ & $7.08 E-02$ & $5.72 E-04$ & -3.19 & $5.57 E-02$ & $5.48 E-02$ & $4.41 E-04$ & -1.51 \\
\hline & 244.69 & $5.68 E-02$ & $5.60 E-02$ & $4.64 E-04$ & -1.50 & $4.36 E-02$ & $4.38 E-02$ & $3.20 E-04$ & 0.37 \\
\hline & 344.28 & $4.53 E-02$ & $4.53 E-02$ & $3.49 E-04$ & 0.12 & $3.50 E-02$ & $3.52 E-02$ & $2.66 E-04$ & 0.52 \\
\hline & 778.90 & $2.04 E-02$ & $2.09 E-02$ & $1.71 E-04$ & 2.60 & $1.60 E-02$ & $1.64 E-02$ & $1.22 E-04$ & 2.59 \\
\hline & 964.13 & $1.58 E-02$ & $1.63 E-02$ & $1.38 E-04$ & 3.13 & $1.24 E-02$ & $1.28 E-02$ & $9.85 E-05$ & 3.20 \\
\hline & 1408.01 & $1.08 E-02$ & $1.13 E-02$ & $8.65 E-05$ & 4.14 & $8.60 E-03$ & $8.85 E-03$ & $6.48 E-05$ & 2.82 \\
\hline \multirow{2}{*}{ Nuclide } & Energy & \multicolumn{4}{|c|}{$\mathcal{E}_{\mathrm{P} 8} \rightarrow \varepsilon_{(\mathrm{V} 1-\mathrm{P} 0)}$} & \multicolumn{4}{|c|}{$\varepsilon_{\mathrm{P} 8} \rightarrow \varepsilon_{(\mathrm{V} 2-\mathrm{P} 0)}$} \\
\hline & $\mathrm{keV}$ & Calculated & Measured & Unc. & $\Delta \%$ & Calculated & Measured & Unc. & $\Delta \%$ \\
\hline \multirow{6}{*}{ Eu-152 } & 121.78 & $7.36 E-02$ & $7.08 E-02$ & $5.72 E-04$ & -3.94 & $5.61 E-02$ & $5.48 E-02$ & $4.41 E-04$ & -2.24 \\
\hline & 244.69 & $5.68 E-02$ & $5.60 E-02$ & $4.64 E-04$ & -1.43 & $4.36 E-02$ & $4.38 E-02$ & $3.20 E-04$ & 0.44 \\
\hline & 344.28 & $4.54 E-02$ & $4.53 E-02$ & $3.49 E-04$ & -0.17 & $3.51 E-02$ & $3.52 E-02$ & $2.66 E-04$ & 0.24 \\
\hline & 778.90 & $2.04 E-02$ & $2.09 E-02$ & $1.71 E-04$ & 2.36 & $1.60 E-02$ & $1.64 E-02$ & $1.22 E-04$ & 2.34 \\
\hline & 964.13 & $1.59 E-02$ & $1.63 E-02$ & $1.38 E-04$ & 2.28 & $1.25 E-02$ & $1.28 E-02$ & $9.85 E-05$ & 2.36 \\
\hline & 1408.01 & $1.08 E-02$ & $1.13 E-02$ & $8.65 E-05$ & 4.16 & $8.60 E-03$ & $8.85 E-03$ & $6.48 E-05$ & 2.84 \\
\hline
\end{tabular}

condition, where one must be provided by exact knowledge of the geometrical parameters of the source-detector arrangements taking into account the attenuation of the gammarays by any material between the source and the detector like aluminum end cap, aluminum oxide reflector, and Plexiglas holder. The calculations were based on the efficiency transfer principle and a new straightforward analytical definition to compute the effective solid angle between the parallelepiped source and the detector surface.

\section{Conflict of Interests}

The authors declare that there is no conflict of interests regarding the publication of this paper. 
TABLE 8: Comparison between the measured and calculated full-energy peak efficiencies for parallelepiped sources, V1 and V2, measured at position P1.

\begin{tabular}{|c|c|c|c|c|c|c|c|c|c|}
\hline \multirow{2}{*}{ Nuclide } & \multirow{2}{*}{$\begin{array}{c}\text { Energy } \\
\text { keV }\end{array}$} & \multicolumn{4}{|c|}{$\varepsilon_{\mathrm{P} 4} \rightarrow \varepsilon_{(\mathrm{V} 1-\mathrm{Pl})}$} & \multicolumn{4}{|c|}{$\varepsilon_{\mathrm{P} 4} \rightarrow \varepsilon_{(\mathrm{V} 2-\mathrm{Pl})}$} \\
\hline & & Calculated & Measured & Unc. & $\Delta \%$ & Calculated & Measured & Unc. & $\Delta \%$ \\
\hline \multirow{6}{*}{ Eu-152 } & 121.78 & $2.25 E-02$ & $2.22 E-02$ & $1.80 E-04$ & -0.97 & $2.08 E-02$ & $2.07 E-02$ & $1.67 E-04$ & -0.34 \\
\hline & 244.69 & $1.75 E-02$ & $1.70 E-02$ & $1.38 E-04$ & -2.52 & $1.64 E-02$ & $1.64 E-02$ & $1.35 E-04$ & 0.26 \\
\hline & 344.28 & $1.41 E-02$ & $1.41 E-02$ & $1.08 E-04$ & -0.06 & $1.33 E-02$ & $1.34 E-02$ & $1.10 E-04$ & 0.54 \\
\hline & 778.90 & $6.47 E-03$ & $6.54 E-03$ & $5.45 E-05$ & 1.05 & $6.23 E-03$ & $6.24 E-03$ & $4.95 E-05$ & 0.16 \\
\hline & 964.13 & $5.06 E-03$ & $5.09 E-03$ & $4.26 E-05$ & 0.71 & $4.89 E-03$ & $4.90 E-03$ & $4.05 E-05$ & 0.15 \\
\hline & 1408.01 & $3.49 E-03$ & $3.56 E-03$ & $2.87 E-05$ & 2.12 & $3.39 E-03$ & $3.45 E-03$ & $2.64 E-05$ & 1.72 \\
\hline \multirow{2}{*}{ Nuclide } & Energy & \multicolumn{4}{|c|}{$\varepsilon_{\mathrm{P} 6} \rightarrow \varepsilon_{(\mathrm{Vl}-\mathrm{Pl})}$} & \multicolumn{4}{|c|}{$\varepsilon_{\mathrm{P} 6} \rightarrow \varepsilon_{(\mathrm{V} 2-\mathrm{P} 1)}$} \\
\hline & $\mathrm{keV}$ & Calculated & Measured & Unc. & $\Delta \%$ & Calculated & Measured & Unc. & $\Delta \%$ \\
\hline \multirow{6}{*}{$\mathrm{Eu}-152$} & 121.78 & $2.28 E-02$ & $2.22 E-02$ & $1.80 E-04$ & -2.72 & $2.11 E-02$ & $2.07 E-02$ & $1.67 E-04$ & -2.08 \\
\hline & 244.69 & $1.74 E-02$ & $1.70 E-02$ & $1.38 E-04$ & -2.42 & $1.64 E-02$ & $1.64 E-02$ & $1.35 E-04$ & 0.35 \\
\hline & 344.28 & $1.40 E-02$ & $1.41 E-02$ & $1.08 E-04$ & 0.81 & $1.32 E-02$ & $1.34 E-02$ & $1.10 E-04$ & 1.41 \\
\hline & 778.90 & $6.34 E-03$ & $6.54 E-03$ & $5.45 E-05$ & 2.94 & $6.11 E-03$ & $6.24 E-03$ & $4.95 E-05$ & 2.07 \\
\hline & 964.13 & $4.92 E-03$ & $5.09 E-03$ & $4.26 E-05$ & 3.41 & $4.76 E-03$ & $4.90 E-03$ & $4.05 E-05$ & 2.87 \\
\hline & 1408.01 & $3.39 E-03$ & $3.56 E-03$ & $2.87 E-05$ & 4.80 & $3.30 E-03$ & $3.45 E-03$ & $2.64 E-05$ & 4.40 \\
\hline \multirow{2}{*}{ Nuclide } & Energy & \multicolumn{4}{|c|}{$\varepsilon_{\mathrm{P} 8} \rightarrow \varepsilon_{(\mathrm{V} 1-\mathrm{Pl})}$} & \multicolumn{4}{|c|}{$\varepsilon_{\mathrm{P} 8} \rightarrow \varepsilon_{(\mathrm{V} 2-\mathrm{P} 1)}$} \\
\hline & Ke & Calculated & Measured & Unc. & $\Delta \%$ & Calculated & Measured & Unc. & $\Delta \%$ \\
\hline \multirow{6}{*}{$\mathrm{Eu}-152$} & 121.78 & $2.30 E-02$ & $2.22 E-02$ & $1.80 E-04$ & -3.46 & $2.13 E-02$ & $2.07 E-02$ & $1.67 E-04$ & -2.82 \\
\hline & 244.69 & $1.74 E-02$ & $1.70 E-02$ & $1.38 E-04$ & -2.35 & $1.64 E-02$ & $1.64 E-02$ & $1.35 E-04$ & 0.43 \\
\hline & 344.28 & $1.40 E-02$ & $1.41 E-02$ & $1.08 E-04$ & 0.53 & $1.33 E-02$ & $1.34 E-02$ & $1.10 E-04$ & 1.13 \\
\hline & 778.90 & $6.36 E-03$ & $6.54 E-03$ & $5.45 E-05$ & 2.70 & $6.13 E-03$ & $6.24 E-03$ & $4.95 E-05$ & 1.82 \\
\hline & 964.13 & $4.96 E-03$ & $5.09 E-03$ & $4.26 E-05$ & 2.57 & $4.80 E-03$ & $4.90 E-03$ & $4.05 E-05$ & 2.02 \\
\hline & 1408.01 & $3.39 E-03$ & $3.56 E-03$ & $2.87 E-05$ & 4.82 & $3.30 E-03$ & $3.45 E-03$ & $2.64 E-05$ & 4.42 \\
\hline
\end{tabular}

\section{Acknowledgment}

The authors would like to express their gratitude for Professor Dr. Marie Christine Lépy, Laboratoire National Henri Becquerel-CEA (LNHB-CEA), Saclay, France, for the fruitful discussions.

\section{References}

[1] M. J. Vargas, A. F. Timón, N. C. Díaz, and D. P. Sánchez, “Monte Carlo simulation of the self-absorption corrections for natural samples in gamma-ray spectrometry," Applied Radiation and Isotopes, vol. 57, no. 6, pp. 893-898, 2002.

[2] M. Korun, "Measurement of the average path length of gammarays in samples using scattered radiation," Applied Radiation and Isotopes, vol. 56, no. 1-2, pp. 77-83, 2002.

[3] J.-M. Laborie, G. Le Petit, D. Abt, and M. Girard, "Monte Carlo calculation of the efficiency calibration curve and coincidencesumming corrections in low-level gamma-ray spectrometry using well-type HPGe detectors," Applied Radiation and Isotopes, vol. 53, no. 1-2, pp. 57-62, 2000.

[4] L. Moens and J. Hoste, "Calculation of the peak efficiency of high-purity germanium detectors," The International Journal of Applied Radiation and Isotopes, vol. 34, no. 8, pp. 1085-1095, 1983.

[5] L. Moens, J. De Donder, X.-L. Lin et al., "Calculation of the absolute peak efficiency of gamma-ray detectors for different counting geometries," Nuclear Instruments and Methods in Physics Research, vol. 187, no. 2-3, pp. 451-472, 1981.

[6] M. S. Badawi, I. Ruskov, M. M. Gouda et al., "A numerical approach to calculate the full-energy peak efficiency of HPGe well-type detectors using the effective solid angle ratio," Journal of Instrumentation, vol. 9, no. 7, Article ID P07030, 2014.

[7] M. S. Badawi, M. Abd-Elzaher, A. A. Thabet, and A. M. ElKhatib, "An empirical formula to calculate the full energy peak efficiency of scintillation detectors," Applied Radiation and Isotopes, vol. 74, pp. 46-49, 2013.

[8] Y. S. Selim and M. I. Abbas, "Direct calculation of the total efficiency of cylindrical scintillation detectors for non-axial point sources," Egyptian Journal of Physics, vol. 26, no. 1-2, p. 79, 1995.

[9] M. S. Badawi, A. M. El-Khatib, and M. E. Krar, "New numerical simulation approach to calibrate the $\mathrm{NaI}(\mathrm{Tl})$ detectors array using non-axial extended spherical sources," Journal of Instrumentation, vol. 8, no. 11, Article ID P11005, 2013.

[10] A. Hamzawy, "Simple analytical formula to calculate $\gamma$-ray cylindrical detectors efficiencies," Nuclear Instruments and Methods in Physics Research Section A: Accelerators, Spectrometers, Detectors and Associated Equipment, vol. 624, no. 1, pp. 125-129, 2010.

[11] Y. S. Selim and M. I. Abbas, "Direct calculation of the total efficiency of cylindrical scintillation detectors for extended circular sources," Radiation Physics and Chemistry, vol. 48, no. 1, pp. 23-27, 1996. 
[12] A. M. El-Khatib, A. A. Thabet, M. A. Elzaher, M. S. Badawi, and B. A. Salem, "Study on the effect of the self-attenuation coefficient on $\gamma$-ray detector efficiency calculated at low and high energy regions," Nuclear Engineering and Technology, vol. 46, no. 2, pp. 217-224, 2014.

[13] M. S. Badawi, M. E. Krar, A. M. El-Khatib, S. I. Jovanovic, A. D. Dlabac, and N. N. Mihaljevic, "A new mathematical model for determining the full energy peak efficiency (FEPE) for an array of two $\gamma$-detectors counting rectangular parallelepiped source," Nuclear Technology \& Radiation Protection, vol. 28, no. 4, pp. 370-380, 2013.

[14] A. M. El-Khatib, M. E. Krar, and M. S. Badawi, "Studying the full energy peak efficiency for a two $\gamma$-detectors combination of different dimensions," Nuclear Instruments and Methods in Physics Research Section A: Accelerators, Spectrometers, Detectors and Associated Equipment, vol. 719, pp. 50-56, 2013.

[15] A. M. El-Khatib, M. M. Gouda, M. S. Badawi, S. S. Nafee, and E. A. El-Mallah, "New analytical approach to calibrate the NaI (Tl) detectors using spherical radioactive sources," Radiation Protection Dosimetry, vol. 156, no. 1, pp. 109-117, 2013. 


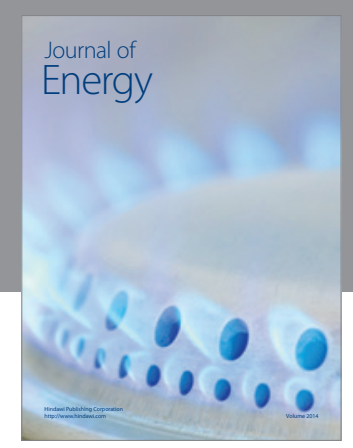

Journal of

Industrial Engineering
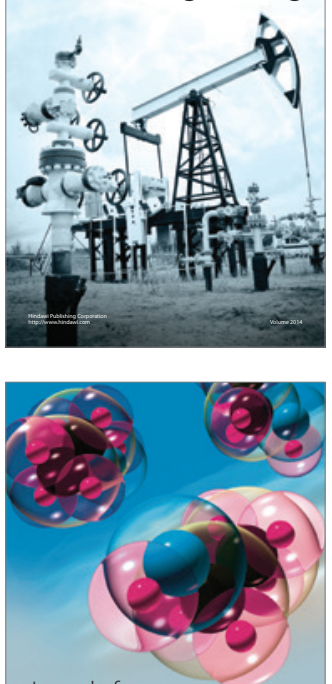

Fuels
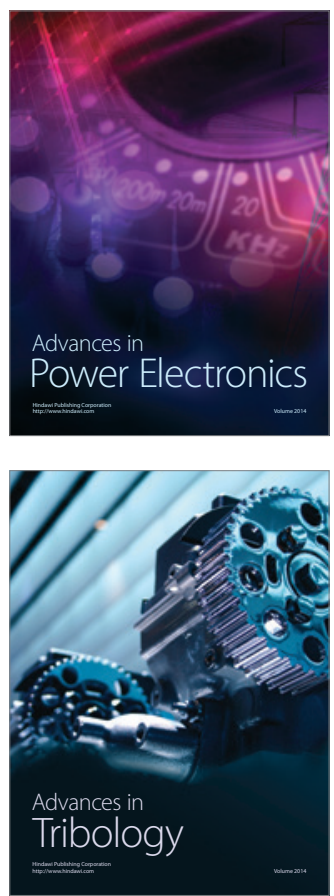

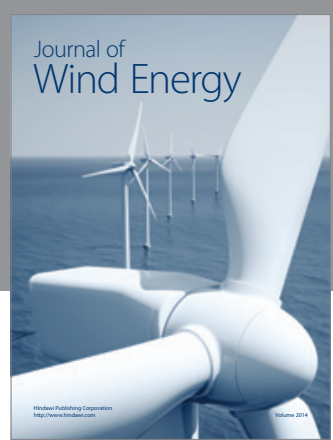

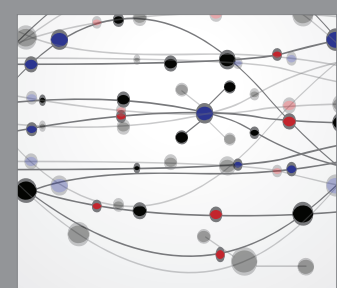

The Scientific World Journal

Submit your manuscripts at http://www.hindawi.com

Journal of

Structures
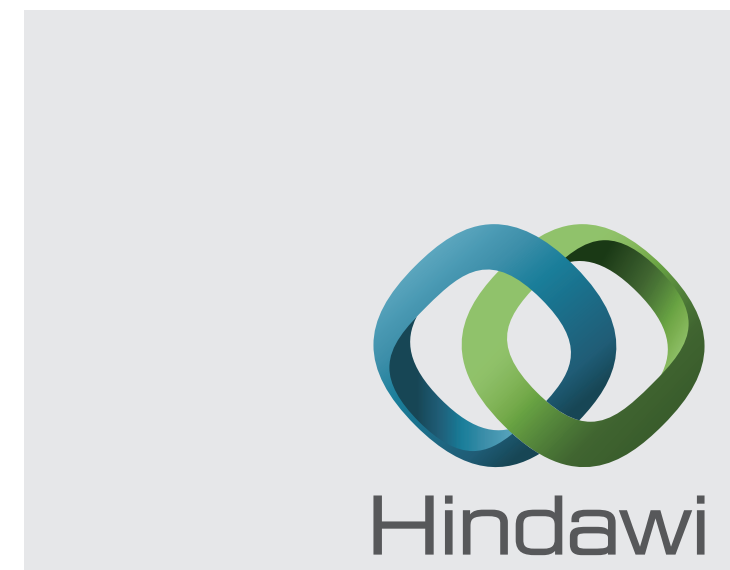

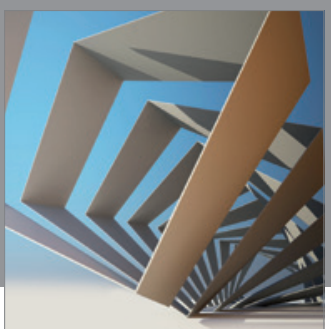

Rotating

Machinery
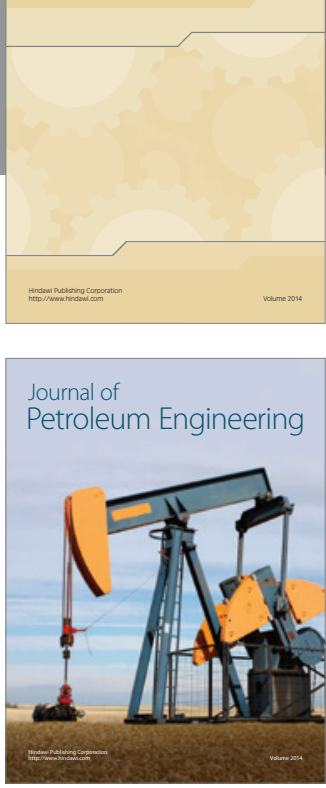

Journal of

Solar Energy
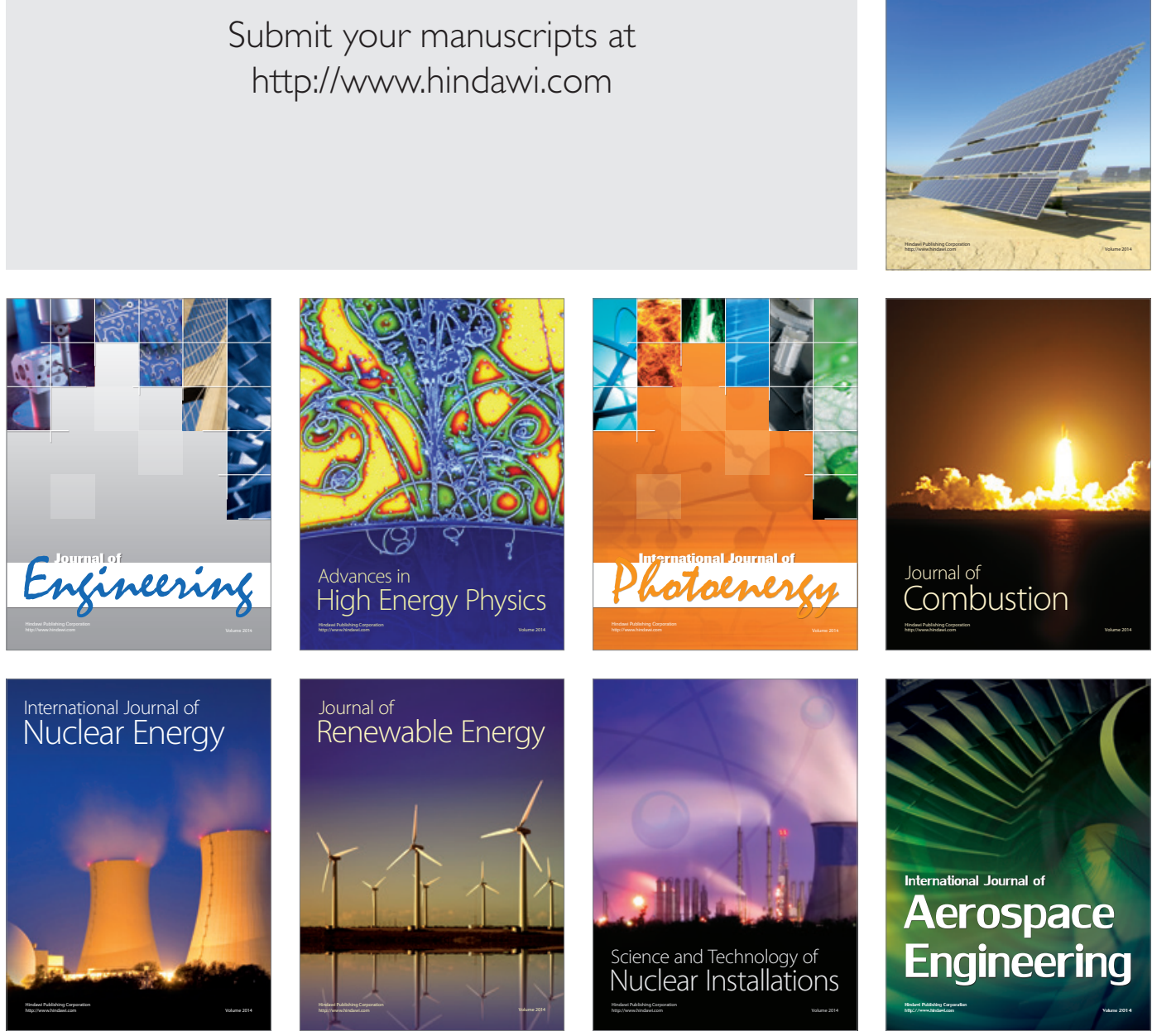